\title{
dATF4 regulation of mitochondrial folate-mediated one-carbon metabolism is neuroprotective
}

\author{
Ivana Celardo ${ }^{1,2}$, Susann Lehmann ${ }^{1,2}$, Ana C Costa ${ }^{1,2}$, Samantha HY Loh ${ }^{\star, 1}$ and L Miguel Martins ${ }^{\star, 1}$
}

Neurons rely on mitochondria as their preferred source of energy. Mutations in PINK1 and PARKIN cause neuronal death in early-onset Parkinson's disease (PD), thought to be due to mitochondrial dysfunction. In Drosophila pink1 and parkin mutants, mitochondrial defects lead to the compensatory upregulation of the mitochondrial one-carbon cycle metabolism genes by an unknown mechanism. Here we uncover that this branch is triggered by the activating transcription factor 4 (ATF4). We show that ATF4 regulates the expression of one-carbon metabolism genes SHMT2 and NMDMC as a protective response to mitochondrial toxicity. Suppressing Shmt2 or Nmdmc caused motor impairment and mitochondrial defects in flies. Epistatic analyses showed that suppressing the upregulation of Shmt2 or Nmdmc deteriorates the phenotype of pink1 or parkin mutants. Conversely, the genetic enhancement of these one-carbon metabolism genes in pink1 or parkin mutants was neuroprotective. We conclude that mitochondrial dysfunction caused by mutations in the Pink1/Parkin pathway engages ATF4-dependent activation of one-carbon metabolism as a protective response. Our findings show a central contribution of ATF4 signalling to PD that may represent a new therapeutic strategy. A video abstract for this article is available at https://youtu.be/cFJJm2YZKKM.

Cell Death and Differentiation (2017) 24, 638-648; doi:10.1038/cdd.2016.158; published online 17 February 2017

Eukaryotic cells respond to diverse stress signals with nuclear gene expression-designed programmes to repair cellular damage or induce apoptosis. Integration of several forms of cellular stress such as amino-acid limitation, endoplasmic reticulum (ER) stress, introduction of double-stranded RNAs into cells by viral infection and heme limitation are transduced via elF2 $a$ kinases. Phospho-elF2a suppresses general protein synthesis, but promotes a paradoxical increase in translation of selected mRNA species such as the activating transcription factor 4 (ATF4) (reviewed in Kilberg et al. ${ }^{1}$ ). Mammalian ATF4, in turn, activates downstream transcriptional programmes designed to induce a cellular adaptation to stress. ATF4 signalling regulates a wide array of genes involved in the response to amino-acid imbalance ${ }^{2,3}$ as well as redox enzymes.

Some autosomal recessive forms of Parkinson's (PD) disease are caused by mutations in PINK1 or PARKIN, two genes involved in mitochondrial quality control (reviewed in Celardo et al. $^{4}$ ). In Drosophila melanogaster, mutations in either pink1 or parkin cause mitochondrial dysfunction linked to the accumulation of defective organelles. Studies in this fly model showed that mitochondrial stress in pink1 mutant results in the transcriptional activation of mitochondrial folatemediated one-carbon metabolism genes as a protective response through an unknown mechanism. ${ }^{5}$ Folate onecarbon metabolism shuttles one-carbon units for biosynthetic pathways, including nucleotide biosynthesis and methylation reactions. Enhancing one-carbon metabolism by exogenous administration of folic acid rescued mitochondrial defects in both flies and cultured human cells. ${ }^{5}$ The mitochondrial one- carbon metabolism was remodelled following mitochondrial dysfunction caused by defects in the replication of mitochondrial DNA (mtDNA) in mice. ${ }^{6}$

One-carbon metabolism (reviewed in Tibbetts and Appling ${ }^{7}$ ) comprises two parallel pathways: one in the cytosol and one in mitochondria. Mitochondrial serine hydroxymethyl transferase (SHMT2) converts serine into glycine and a formyl unit attached to tetrahydrofolate (THF) that is further converted to 10-formyl-THF by NAD-dependent methylenetetrahydrofolate dehydrogenase (NMDMC), also known as mitochondrial methylenetetrahydrofolate dehydrogenase (MTHFD2). 10-formyl-THF is required to make formylmethionine for mitochondrial protein synthesis. ${ }^{8}$ These mitochondrial onecarbon metabolism enzymes are essential for both embryonic development ${ }^{9}$ and tumourigenesis, ${ }^{10}$ and both SHMT2 and NMDMC are consistently upregulated in rapidly proliferating cancer cells. ${ }^{11-13}$

We found that ATF4 controls the expression of the mitochondrial one-carbon metabolism genes SHMT2 and NMDMC as a protective response to mitochondrial toxicity. In vivo RNAi-mediated downregulation of Shmt2 or Nmdmc caused mitochondrial impairment. Conversely, their genetic enhancement suppressed neurodegeneration in both pink1 and parkin mutant flies.

We conclude that mitochondrial dysfunction following disruption of the Pink1/Parkin pathway can be suppressed by the genetic enhancement of mitochondrial one-carbon metabolism. This shows that the one-carbon metabolism pathway is not only critical for the survival of proliferating cells such as cancer cells but also sustains

${ }^{1}$ MRC Toxicology Unit, Lancaster Road, Leicester LE1 9HN, UK

${ }^{*}$ Corresponding author: LM Martins or SHY Loh, Cell Death Regulation Laboratory, MRC Toxicology Unit, Hodgkin Building, Lancaster Road, Leicester LE1 9HN, UK.

Tel: +(0)44 1162525533 or +(0)44 116223 1501; Fax: +44 1162525616 or +44 116252 5616; E-mail: martins.Imiguel@gmail.com or shyl1@le.ac.uk

${ }^{2}$ These authors contributed equally to this work.

Received 08.7.16; revised 01.12.16; accepted 12.12.16; Edited by L Greene; published online 17.2.2017 
a

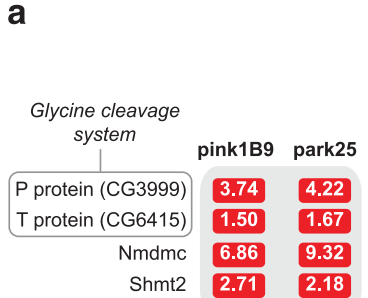

b

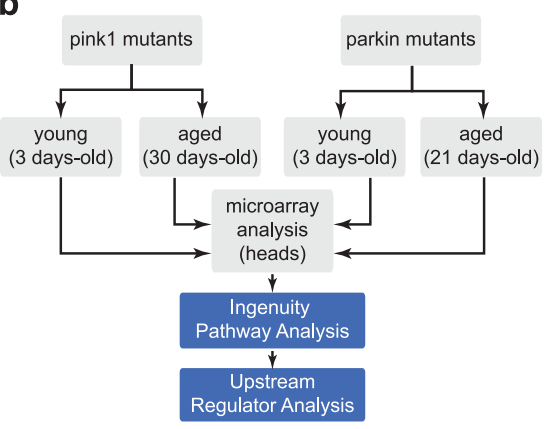

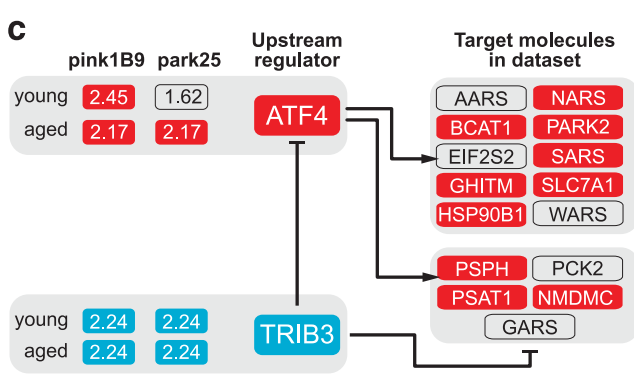

d

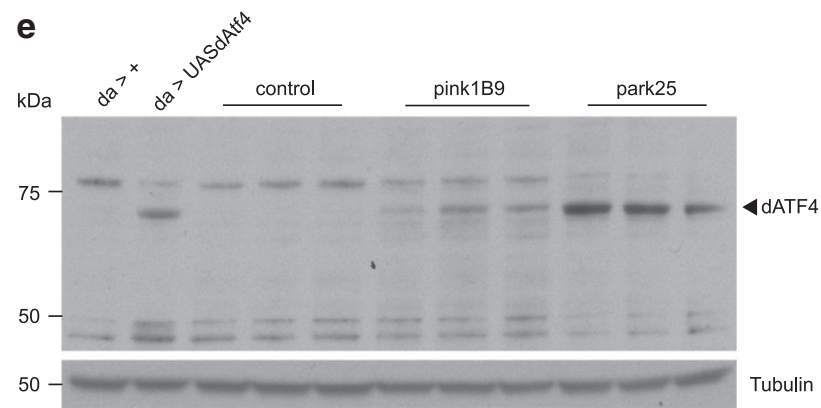

Figure 1 In silico identification of ATF4 as a regulator of transcriptional changes in pink1 and parkin mutants. (a) Enhanced expression of one-carbon metabolism genes Shmt2, Nmdmc, CG3999 (GCS P protein, glycine dehydrogenase) and CG6415 (GCS T protein, aminomethyl transferase) in heads of 3-day-old pink1 or parkin mutant flies. Relative levels to the $w^{1118}$ control flies are indicated. Red corresponds to transcripts that are upregulated to a significant $(P \leqslant 0.05)$ level. Significance was determined using a two-tailed unpaired $t$-test ( 3 to 8 biological replicates per sample). (b) Workflow employed for the identification of upstream regulators of transcriptional changes upon loss of pink 1 or parkin. (c) Analysis of upstream modulators of gene expression changes observed in pink1 (pink $1^{\text {B9}}$ ) and parkin (park ${ }^{25}$ ) mutant flies. The term 'young' corresponds to heads from 3-day-old pink1 and parkin mutant flies and 'aged' to heads from 21- and 30-day-old parkin and pink1 mutant flies, respectively. The activation z-scores for ATF4 and TRIB3 are indicated. Red and blue correspond to significant positive $(z \geqslant 2)$ and negative $(z \leqslant-2)$ scores, respectively. Target molecules labelled in red correspond to positive transcripts regulated in the analysed data sets. (d) Changes in amino-acid abundance upon the loss of pink1 or parkin function. Relative levels to the $w^{1118}$ control flies are indicated. Red and blue correspond to metabolites that are upregulated and downregulated to a significant level, respectively. ND corresponds to an amino-acid below detection threshold. Significance was determined using Welch's two-sample $t$-test $(n=8)$. (e) Analysis of dAtf4 protein levels. Whole-fly lysates were analysed by western blotting using the indicated antibodies. The first two lanes correspond to a control for the specificity of the anti-dAtt4 antibody performed by analysing the levels of overexpressed dAtt 4 in transgenic flies (da > UAS dAtt4). Genotypes: da >+, daGal4 > +; da > UASdAtt4, daGal4 > UASdAtt4; control, $w^{1118}$

the viability of post-mitotic cells such as neurons by promoting mitochondrial health.

\section{Results}

Identification of ATF4 as an upstream regulator of onecarbon metabolism genes in pink1 and parkin mutants. We have previously observed an upregulation of nucleotide metabolism pathways, including the one-carbon metabolism enzymes, in the heads of Drosophila pink1 (pink $1^{B 9}$ ) mutants flies. ${ }^{5}$ As Parkin cooperates with Pink1 in a pathway involved in mitochondrial quality control (QC), we compared the expression of genes involved in the mitochondrial onecarbon metabolism between pink1 and parkin (park ${ }^{25}$ ) mutants. This revealed a significant increase in mitochondrial transcripts for one-carbon enzymes in the heads of both pink1 and parkin mutants (Figure 1a), indicating that these transcripts are upregulated upon dysfunction of the Pink1/ Parkin mitochondrial QC pathway.

To determine how mitochondrial stress in pink1 and parkin mutants modulates the expression of genes involved in nucleotide metabolism, we explored the mechanism underlying transcriptional changes upon mitochondrial dysfunction. To identify the upstream regulators of altered nuclear gene expression in pink1 or parkin mutants, we employed microarray technology coupled with an in silico approach (experimental outline, Figure 1b). We used Ingenuity upstream regulator analysis, a causal analytics algorithm designed to identify upstream regulators that are connected to data set genes. ${ }^{14}$ This analysis, applied to the full complement of transcriptional changes in either pink1 or parkin mutant heads, revealed a subnetwork of transcriptional changes linked to the upstream triggering of ATF4 as well as to the inhibition of the tribbles pseudokinase 3 (TRB3), a negative feedback regulator of ATF4-dependent transcription, ${ }^{15}$ in both pink1 and parkin mutants (Figure 1c and Supplementary Table 1). We have previously shown that mutations in pink1 or parkin result in a translational shutdown ${ }^{16}$ that normally coincides with an increase in ATF4 activity. ${ }^{17,18}$ As ATF4 activation is linked to amino-acid imbalances, we next measured the endogenous amino-acid levels in pink1 and parkin mutant flies. This metabolic analysis revealed an amino-acid imbalance in both pink1 and parkin mutants, resulting in an increase in the majority of free amino acids $\left(P<0.001, X^{2}\right.$, Figure $\left.1 d\right)$. 
We next assessed the protein levels of dAtf4 in pink1 and parkin mutant flies. This revealed an increase in dAtf4 in both pink1 and parkin adult animals (Figure 1e).

ATF4 is required for the upregulation of SHMT2 and NMDMC upon stress induction. ATF4 mediates the induction of protective genes such as those involved in amino-acid metabolism and oxidative stress. ${ }^{3}$ An analysis of the human orthologues of the mitochondrial one-carbon metabolism genes upregulated in pink1 and parkin mutants using the 'Grow tool' in Ingenuity Pathway Analysis (IPA) suggested that ATF4 is a direct modulator of the SHMT2 and NMDMC transcripts (Figure 2a). To determine the role of $d A T F 4$ in the regulation of Drosophila Shmt2 and Nmdmc, we downregulated its expression using RNA interference (RNAi). dATF4 RNAi resulted in a decrease in the basal transcript levels of Shmt2 and $\mathrm{Nmdmc}$ (Figure 2b), indicating that dATF4 suppression is sufficient to downregulate their expression.

We next tested whether ATF4 also acts as an upstream regulator of SHMT2 and NMDMC in mammalian cells. ATF4 can induce transcriptional changes after toxic insults promoted by classical ER stressors or oxidative stress. ${ }^{19} \mathrm{We}$ treated cultured mammalian cells with thapsigargin, a sarco/ endoplasmic reticulum $\mathrm{Ca}^{2+}$ ATPase (SERCA) inhibitor, or rotenone, a mitochondrial complex I inhibitor. These treatments led to the accumulation of ATF4 (Figures 2c and d) and promoted the transcriptional upregulation of both SHMT2 and NMDMC, as well as a more pronounced upregulation of CHOP (DDIT3), a classical ATF4 target gene, in SH-SY5Y neuroblastoma cells (Figures $2 e$ and f). Following RNAimediated downregulation of ATF4 (Figure $2 \mathrm{~g}$ ), the upregulation of both SHMT2 and NMDMC in cells treated with ATF4activating toxins was blocked (Figures $2 \mathrm{~h}$ and $\mathrm{i}$ ), indicating that ATF4 lies upstream of the activation of both of these one-carbon metabolism transcripts upon ER or mitochondrial stress.

\footnotetext{
Suppression of Drosophila Shmt2 or Nmdmc affects development and causes mitochondrial dysfunction. To investigate the in vivo role of Shmt2 and Nmdmc, we determined the consequences of their suppression using RNAi (Figure 3a). The knockdown of Shmt2 or Nmdmc caused developmental defects characterized by a significant failure of eclosion (Figure 3b). Analysis of the eclosed adults revealed that the knockdown of Shmt2 or Nmdmc resulted in an impaired climbing ability, suggesting a locomotor deficit (Figure 3c), and decreased lifespan (Figure 3d). The knockdown of either Shmt2 or Nmdmc led to significant metabolic changes in several canonical pathways, most significantly those related to nucleotide degradation and salvage (Figure $3 e$ and Supplementary Table 2). To further determine whether the consequences of Shmt2 or Nmdmc knockdown were linked to mitochondrial defects, we performed a morphological and functional analysis of mitochondria. This revealed a fragmented mitochondrial network (Figures $4 a$ and b) that was associated with a loss of mitochondrial membrane potential $(\Delta \psi \mathrm{m})$ in adult brain (Figures $4 \mathrm{c}$ and d) as well as a generalized loss of mitochondrial proteins (Figure 4e). In addition, Nmdmc knockdown adult flies
}

exhibited an abnormal downturned wing posture (Figure 4f) and ultrastructural analysis of their indirect flight muscles revealed mitochondria with fragmented cristae (Figure $4 \mathrm{~g}$ ).

Taken together, these data suggest that the loss of the mitochondrial one-carbon metabolism genes Shmt2 and $\mathrm{Nmdmc}$ results in mitochondrial dysfunction.

Suppressing the upregulation of Shmt2 or Nmdmc deteriorates the PD-linked phenotypes in pink1 and parkin mutants. To confirm that transcripts for the onecarbon metabolism genes Shmt2 and $\mathrm{Nmdmc}$ are induced in pink1 and parkin mutants as a compensatory mechanism for mitochondrial dysfunction, we tested the consequences of their suppression in these mutants. As ATF4 is required for the upregulation of SHMT2 and NMDMC in mammalian cells, we first determined the in vivo consequences of suppressing its expression in pink 1 or parkin mutant flies. The knockdown of $d$ Atf4 led to $11 \%$ and $84 \%$ lethality, respectively, in pink1 and parkin mutants (Figure 5a). Analysis of the surviving pink1 and parkin mutant adults with dAtf4 knockdown confirmed the downregulation of the dAtf4 transcript (Figure $5 \mathrm{~b}$ ) and revealed an increased penetrance of the crushed-thorax phenotype (Figures $5 \mathrm{c}$ and $\mathrm{d}$ ). In addition, dAtf4 knockdown caused a decline in the climbing performance of pink1 mutants (Figure 5e). We next assessed the effect of $d A t f 4$ knockdown on the levels of Shmt2 and Nmdmc in pink1 and parkin mutants. This caused a moderate decrease in Shmt2 and a more pronounced decrease in $\mathrm{Nmdmc}$ transcript levels (Figures $5 \mathrm{f}$ and $\mathrm{g}$ ). Taken together, these results show that dAtf4 is involved in the upregulation of Shmt2 and Nmdmc in pink1 and parkin mutants. Next, we tested the effects of downregulating Shmt2 or Nmdmc in pink1 or parkin mutants. The knockdown of Shmt2 or Nmdmc led to $100 \%$ and $99 \%$ lethality, respectively, in parkin mutants (Figure 5h). In pink1 mutants, the knockdown of Shmt2 or $\mathrm{Nmdmc}$ reduced their respective transcript levels (Figure $5 \mathrm{i}$ ), and caused 84 and 19\% lethality, respectively (Figure 5j). Analysis of the surviving adults revealed an increased penetrance of the crushed-thorax phenotype in both Shmt2 and $\mathrm{Nmdmc}$ knockdown (Figure 5k). Furthermore, Nmdmc knockdown exhibited a dramatic decline in the climbing performance of pink1 mutants (Figure 5l). These results suggest that the one-carbon metabolism genes, Shmt2 and $\mathrm{Nmdmc}$, were upregulated as a protective mechanism in pink1 and parkin mutants.

Expression of Shmt2 or Nmdmc rescues the PD-linked phenotypes in pink1 and parkin mutants. We previously showed that the pharmacological enhancement of folic acid metabolism confers a protective effect on the mitochondria of pink1 or parkin mutants. ${ }^{5}$ We therefore examined the effects of overexpressing one-carbon metabolism genes, Shmt2 and $\mathrm{Nmdmc}$, in pink1 or parkin mutants. We first confirmed the overexpression of either Shmt2 or Nmdmc (Figures 6a and b) in adult flies. Next, by targeting the expression of these genes, using a neuronal driver, we rescued the mitochondrial function in neurons (Figure $6 \mathrm{c}$ ) and the loss of dopaminergic neurons in the PPL1 cluster of both pink1 and parkin mutants (Figures 6d-f). Taken together, these results show that the one-carbon metabolism genes have a neuroprotective role in 
the Pink1/Parkin PD model. We showed that Shmt2 and Nmdmc expression is in part mediated by ATF4 in pink 1 and parkin mutants (Figures $5 f$ and $g$ ). ATF 4 can be activated by the ER stress-inducible kinase PERK and Gcn2, a kinase that acts as a sensor of amino-acid depletion by binding uncharged tRNAs and phosphorylating elF2a on serine 51

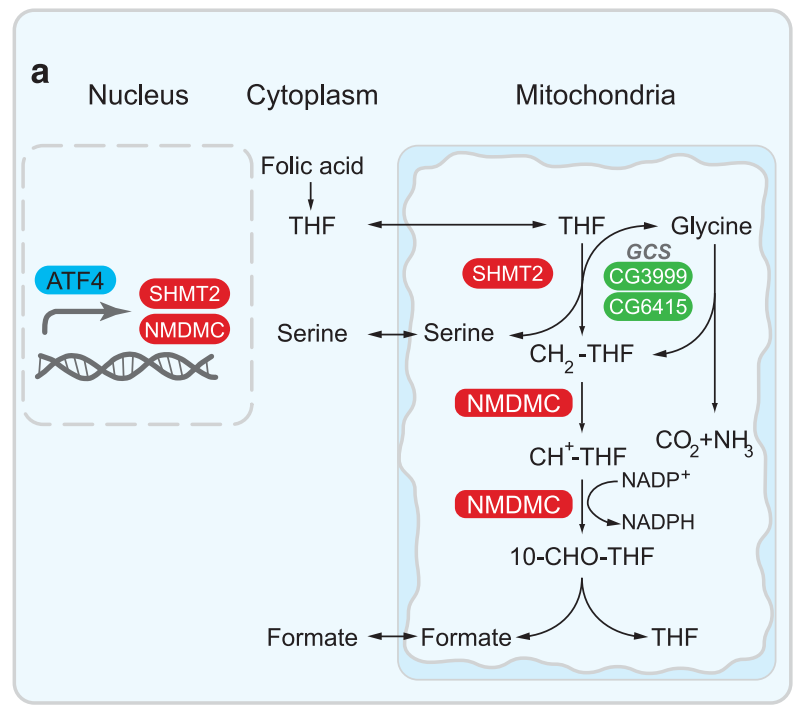

b

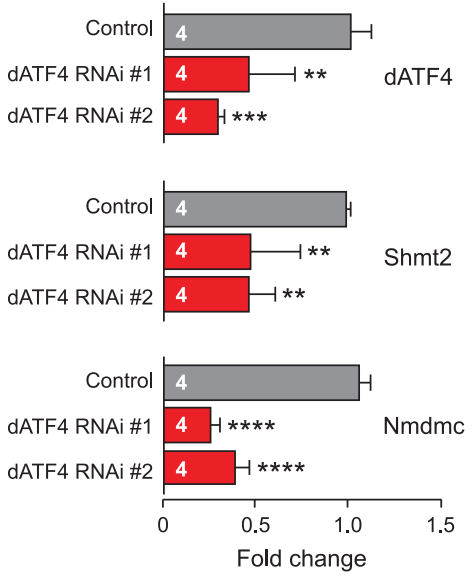

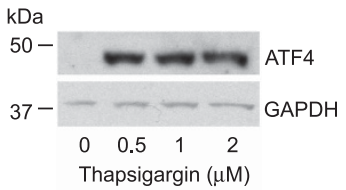

e

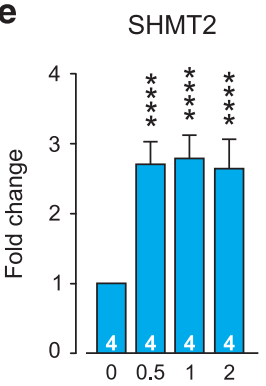

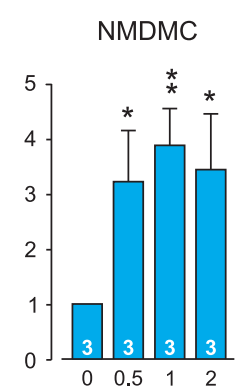

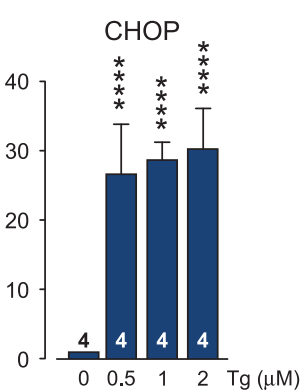

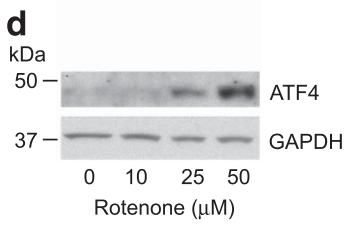

g

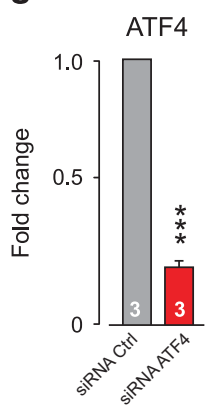

h
SHMT2

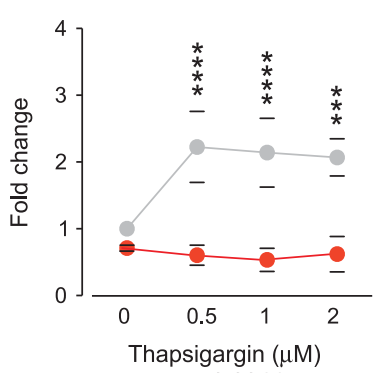

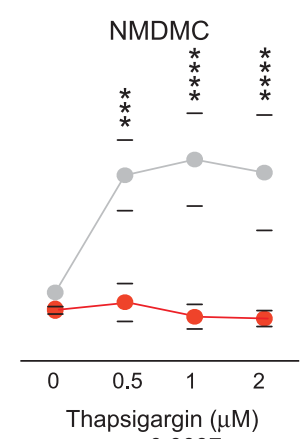

$p=0.0037$ $\mathbf{f}$

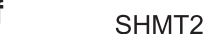

$\mathrm{CHOP}$

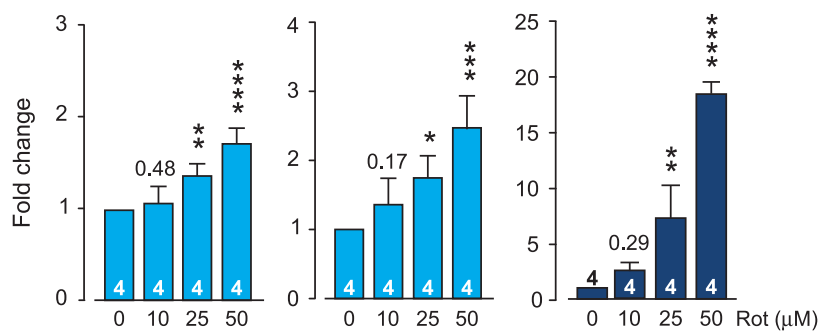

Figure 2 ATF4 regulates the expression of Shmt2 and Nmdmc. (a) In silico identification of SHMT2 and NMDMC (red) as direct downstream transcriptional targets of ATF4. ATF4 could be directly connected to SHMT2 and NMDMC using the 'Grow tool' in IPA. The other one-carbon metabolism genes, CG3999 (GCS P protein, glycine dehydrogenase) and CG6415 (GCS T protein, aminomethyl transferase) (green), found to be upregulated in the heads of pink1 or parkin mutant flies are also shown. A schematic diagram of metabolites related to these enzymes is also shown. (b) dATF4 RNAi flies show a downregulation of mitochondrial Shmt2 and Nmdmc (mean \pm S.D.; asterisks, one-way ANOVA with Bonferroni's multiple comparison test). Genotypes: Control: elavGAL4, all RNAi were driven by elavGAL4. (c and d) Increased levels of ATF4 following toxin-induced ER or mitochondrial stress. SH-SY5Y cells were incubated for $8 \mathrm{~h}$ in the presence of the indicated concentrations of the ER stressor thapsigargin (c) or the complex I inhibitor rotenone (d). Cell lysates were analysed using the indicated antibodies. (e and f) Transcriptional upregulation of SHMT2, NMDMC and CHOP following ER or mitochondrial stress (mean \pm S.D.; asterisks, one-way ANOVA with Bonferroni's multiple comparison test). SH-SY5Y cells were incubated for $8 \mathrm{~h}$ in the presence of the indicated concentrations of thapsigargin (Tg) (e) or rotenone (Rot) (f). (g) Downregulation of ATF4 in SH-SY5Y cells. The fold change of the indicated transcript levels compared with control siRNA is indicated (mean \pm S.D.; asterisks, one-tailed unpaired $t$-test relative to control). ( $h$ and i) The siRNA-mediated downregulation of ATF4 blocks the toxin-induced transcriptional upregulation of SHMT2 and NMDMC. Analysis of SHMT2 and NMDMC transcript levels in ATF4 RNAi cells following toxin treatment. SH-SY5Y cells were incubated for $8 \mathrm{~h}$ in the presence of the indicated concentrations of thapsigargin $(\mathrm{Tg})(\mathrm{h})$ or rotenone (Rot) (i) (mean \pm S.D., $n=3$; asterisks, one-way ANOVA for the siRNA variable with Bonferroni's multiple comparison test, $P$-value for the siRNA-drug interaction two-way ANOVA is also indicated) 
(reviewed in Kilberg et al. ${ }^{1}$ ). We showed that suppression of protein kinase R-like endoplasmic reticulum kinase (PERK) is neuroprotective in pink1 and parkin mutants. ${ }^{16}$ As we observed significant alterations in amino-acid levels in pink1 and parkin mutants (Figure 1d), we tested whether Drosophila Gcn2 (dGcn2) also plays a role in the activation of ATF4 in these mutants. RNAi-mediated suppression of dGcn2 failed to rescue the neuronal loss in pink1 or parkin mutant a

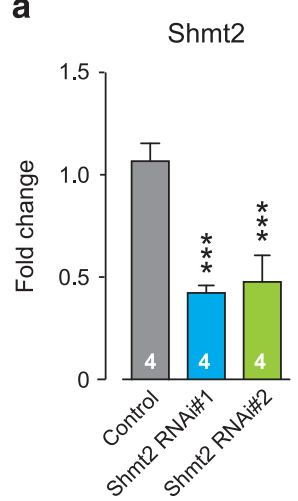

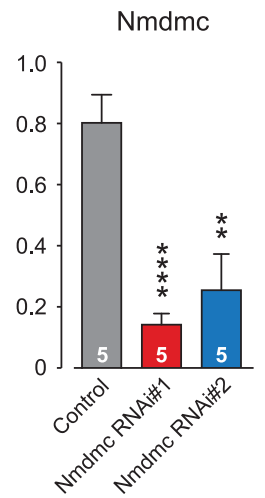

b

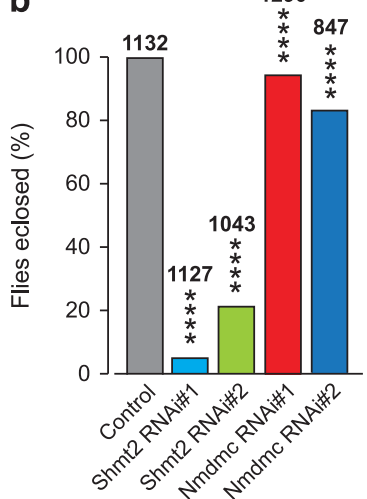

C

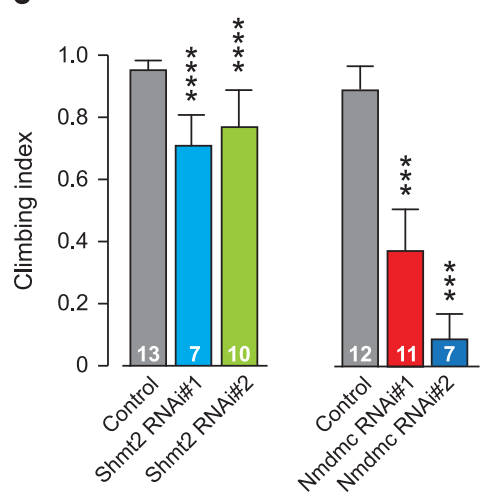

d
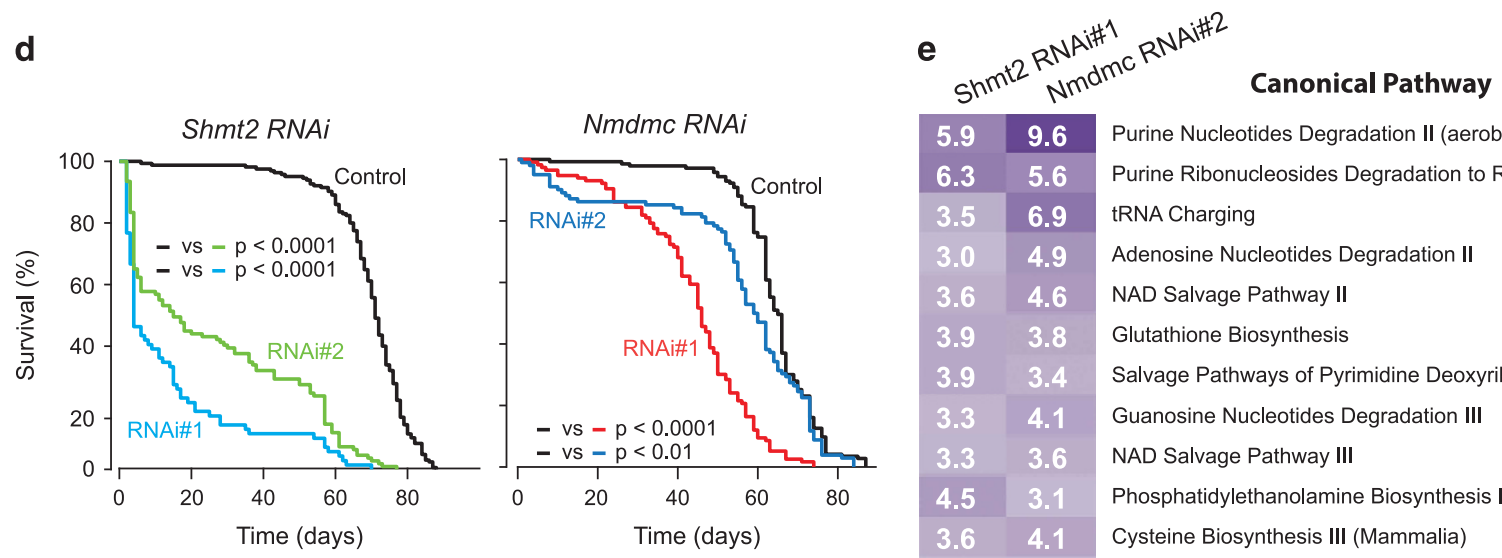

\begin{tabular}{|l|l|l|}
\hline 5.9 & 9.6 & Purine Nucleotides Degradation II (aerobic) \\
\hline 6.3 & 5.6 & Purine Ribonucleosides Degradation to Ribose-1-phosphate \\
\hline 3.5 & 6.9 & tRNA Charging \\
3.0 & 4.9 & Adenosine Nucleotides Degradation II \\
3.6 & 4.6 & NAD Salvage Pathway II \\
3.9 & 3.8 & Glutathione Biosynthesis \\
3.9 & 3.4 & Salvage Pathways of Pyrimidine Deoxyribonucleotides \\
3.3 & 4.1 & Guanosine Nucleotides Degradation III \\
3.3 & 3.6 & NAD Salvage Pathway III \\
\hline 4.5 & 3.1 & Phosphatidylethanolamine Biosynthesis II \\
\hline 3.6 & 4.1 & Cysteine Biosynthesis III (Mammalia) \\
3.0 & 3.1 & Citrulline Degradation \\
\hline 3.3 & 3.1 & Adenine and Adenosine Salvage III \\
3.8 & 3.0 & Salvage Pathways of Pyrimidine Ribonucleotides \\
\hline & & \\
\hline
\end{tabular}

Figure 3 In vivo suppression of Shmt2 or Nmdmc affects development and causes motor impairment in adult flies. (a) RNAi-mediated suppression of Shmt2 or Nmdmc. Expression levels were measured by real-time qPCR (mean \pm S.D.). The significant values relative to the control are indicated (asterisks, two-tailed unpaired $t$-test compared with Control). (b) Eclosion defects following RNAi-mediated suppression of Shmt2 or Nmdmc (asterisks, $\chi^{2}$ two-tailed, 95\% confidence intervals). (c) Motor impairment upon RNAimediated suppression of Shmt2 or Nmdmc. Flies were tested using a standard climbing assay (mean \pm S.D.; asterisks, one-way ANOVA with Dunnett's multiple comparison test). (d) Decreased lifespan upon RNAi-mediated suppression of Shmt2 or Nmdmc. Fly viability was scored over a period of 90 days $(n=165$ for Control (left panel), $n=68$ for Shmt2 RNAi\#1, $n=108$ for Shmt2 RNAi\#2, $n=143$ for Control (right panel), $n=116$ for Nmdmc RNAi\#1, $n=102$ for Nmdmc RNAi\#2; asterisks, log-rank, Mantel-Cox test). (e) Coordinated changes in metabolite abundance on the downregulation of Shmt2 or Nmdmc. Canonical pathways altered in both Shmt2 and Nmdmc RNAi flies were analysed using the IPA Comparison Analysis tool. The heat map corresponds to the -log ( $P$-value) for the canonical pathways significantly perturbed by the downregulation of either Shmt2 or Nmdmc (cutoff for $P$-value is 0.001 for both Shmt2 and Nmdmc RNAi data sets). Genotypes in (a-e): Control: daGAL4. All RNAi lines were driven by daGAL4

Figure 4 Suppression of Shmt2 or Nmdmc causes mitochondrial defects. (a and $\mathbf{b})$ RNAi-mediated suppression of Shmt2 or Nmdmc results in mitochondrial fragmentation. (a) Confocal analysis of mitoGFP in the indicated larval tissues. (b) The quantification of mitochondrial length from larval nerve is indicated (mean \pm S.D., $P$-value, one-way ANOVA with Bonferroni's multiple comparison test). (c) Representative confocal image of a whole mounted control brain showing neurons loaded with TMRM. (d) Loss of $\Delta \psi \mathrm{m}$ following RNAi-mediated suppression of Shmt2 or Nmdmc. The data are shown as the mean \pm S.D. (asterisks, one-way ANOVA with Bonferroni's multiple comparison test). (e) Loss of mitochondrial proteins in Shmt2 and Nmdmc RNAi flies. Whole-fly lysates from 3-day-old flies were analysed by western blot analysis using the indicated antibodies. (f) Abnormal wing posture following RNAi-mediated suppression of Nmdmc (asterisks, $\chi^{2}$ two-tailed, $95 \%$ confidence intervals). (g) The knockdown of Nmdmc causes mitochondrial cristae fragmentation. Ultrastructural analysis of the indirect flight muscles in Nmdmc RNAi flies (my, myofibrils; m, mitochondria; yellow outlines, mitochondria). Percentages of indirect-flight-muscle mitochondria exhibiting fragmented cristae normalized to area are presented (asterisks, $\chi^{2}$ two-tailed, $95 \%$ confidence intervals). Genotype in (a and $\mathbf{b}$ ): Control: elavGAL4 > mitoGFP; Shmt2 RNAi \#1: elavGAL4 > mitoGFP, Shmt2 RNAi \#1; Nmdmc RNAi \#1: elavGAL4 > mitoGFP, Nmdmc RNAi \#1, (c and d): Control: elavGAL4. All RNAi lines were driven by elavGAL4, (e-g): Control: daGAL4. All RNAi lines were driven by daGAL4 
flies (Figure 6g). Together, these results indicate that in pink1 and parkin mutants, ATF4 is regulated via PERK.

\section{Discussion}

The mitochondrial retrograde response acts as a sensitive feedback system to fine-tune mitochondrial performance. We have shown that genes affecting key nucleotide metabolic processes alleviate stress through metabolic readjustments that stimulate mitochondrial function and block neurodegeneration. ${ }^{5}$ Furthermore, we reported that mitochondrial dysfunction in pink1 or parkin mutants activates ER stress signalling. ${ }^{16}$ Here, we used an exploratory approach to determine the mechanism underlying the regulation of gene
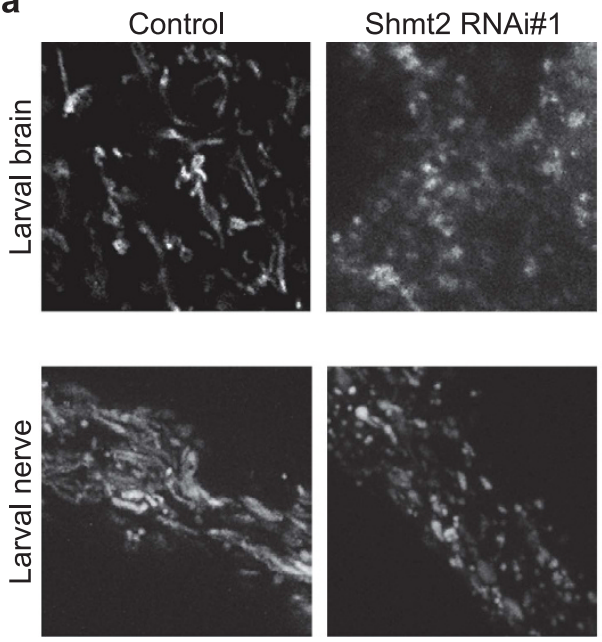

C

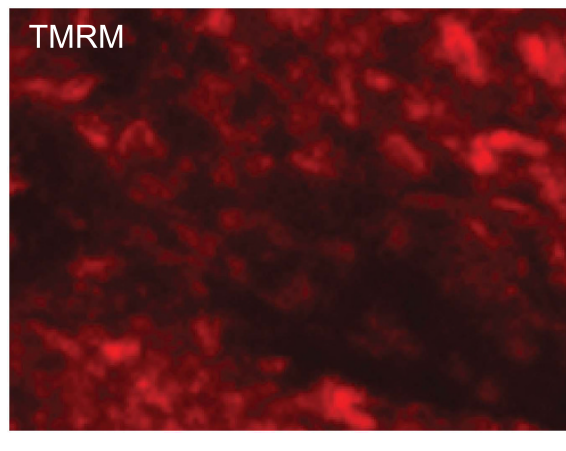

$10 \mu \mathrm{m}$
Nmdmc RNAi\#1
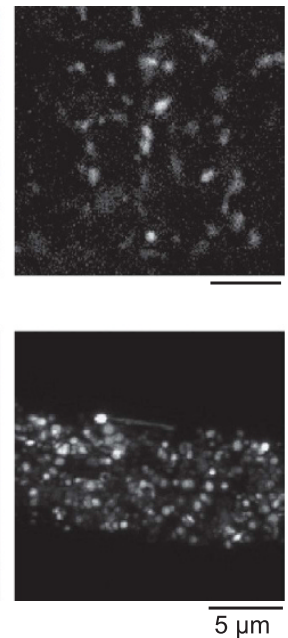

d

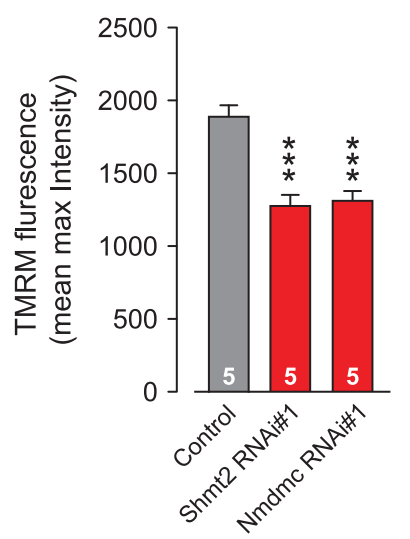

b

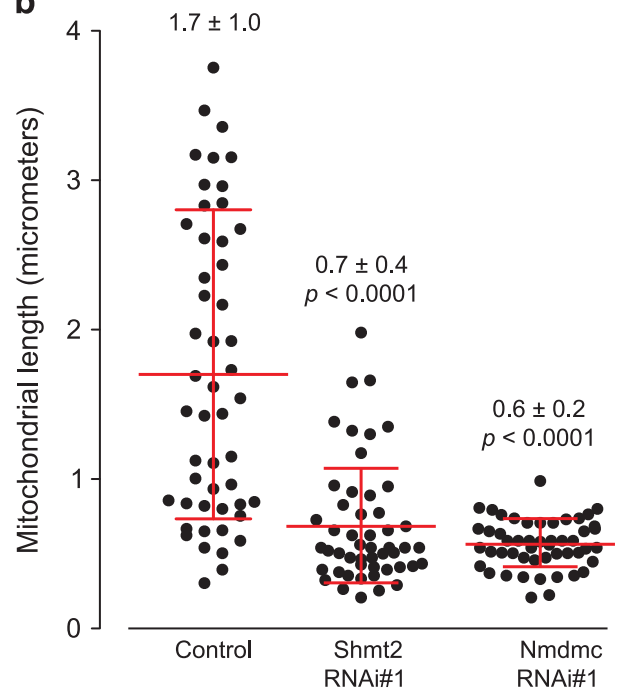

e

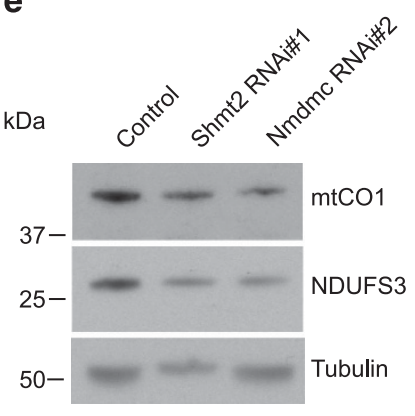

f

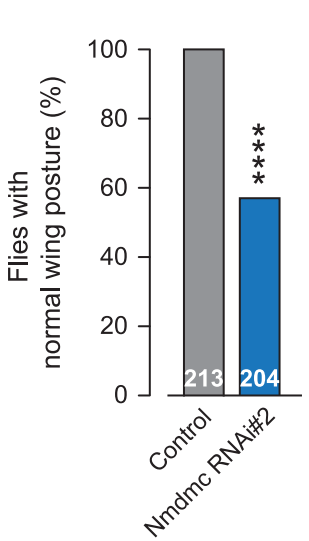

g

Control

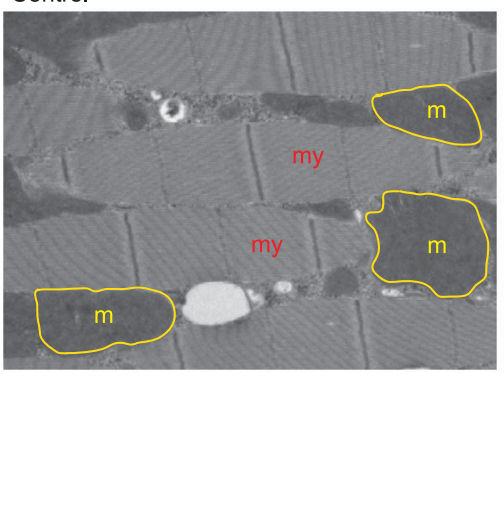

Nmdmc RNAi\#2

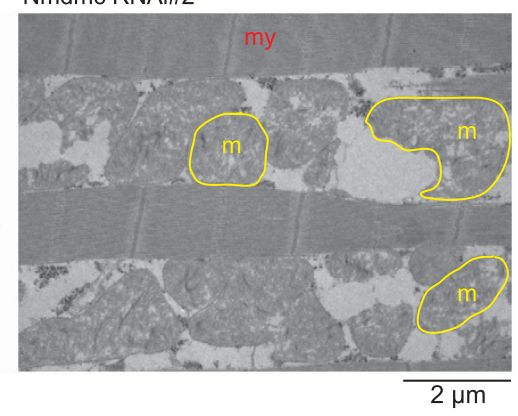

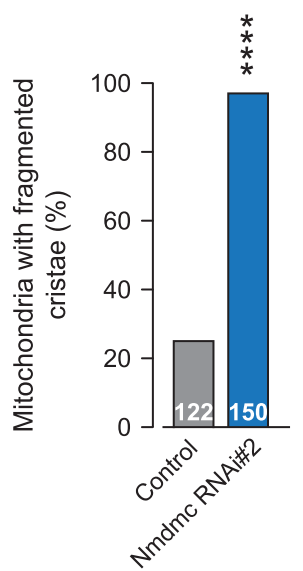


expression following mitochondrial dysfunction in the Pink1/ Parkin pathway. Using a web-based analytical tool, we found evidence for the involvement of ATF4 in the activation of the mitochondrial one-carbon metabolism genes Shmt2 and Nmdmc in pink1 and parkin mutant flies.

We provide evidence that the one-carbon metabolism genes Shmt2 and Nmdmc are under the control of ATF4. Both the ER toxin thapsigargin and the mitochondrial poison rotenone led to the ATF4-dependent activation of SHMT2 and NMDMC, most likely to alleviate stress.

Several cellular stress signals transduced through four elF2 $a$ kinases led to global translation shutdown but also promoted a paradoxical increase in the translation of specific mRNAs, such as that for ATF4 (reviewed in Kilberg et al. ${ }^{1}$ ). We have reported that mitochondrial stress in pink1 or parkin mutant flies results in global translational shutdown via PERK, an elF2 $a$ kinase, with neurotoxic consequences. ${ }^{16}$ In spite of the global translational shutdown, transcripts for Shtm2 and Nmdmc are upregulated in pink1 and parkin mutants (Figures $5 \mathrm{f}$ and $\mathrm{g}$ and Tufi et $a l^{5}{ }^{5}$ ), and here we report that their overexpression via ATF4 is neuroprotective. This illustrates another paradox linked to the activation of PERK. How can PERK signalling cause translational shutdown with a neurotoxic outcome whereas via ATF4 it promotes the activation of neuroprotective genes? We propose that this could result from a compromised biphasic response to PERK activation in neurons. The short-term PERK-dependent translational shutdown in cells is followed by a recovery of translation via GADD34-dependent dephosphorylation of elF2a (reviewed in Kilberg et al. ${ }^{1}$ ). However, the sustained phosphorylation of elF2 $a$ in neurons of pink1 or parkin mutants might not be able to sustain an efficient translation of ATF4 targets even though their mRNA pools are increased.

Eukaryotic one-carbon metabolism comprises parallel cytosolic and mitochondrial pathways connected by the onecarbon donors serine, glycine and formate. We show here that compromising the mitochondrial one-carbon pathway by downregulating Shmt2 or Nmdmc leads to significant changes in nucleotide pathways. Shmt2 or Nmdmc silencing resulted in mitochondrial dysfunction and led to both developmental defects and decreased viability of adult flies. This result suggests that mitochondrial one-carbon metabolism is important for the normal function of these organelles. pink1 or parkin mutants show a significant upregulation of transcripts for Shmt2 and Nmdmc (Figures 5f, $g$ and i). We propose that the enhanced Shmt2 and Nmdmc transcripts levels represents a compensatory attempt to revert the mitochondrial dysfunction caused by defects in the Pink1/Parkin pathway, as the rescue of neurodegeneration in either pink1 or parkin mutants can be achieved by the overexpression of either Shmt2 or Nmdmc. If both Shmt2 and Nmdmc are already upregulated in pink1 or parkin mutants, why then do we need to artificially enhance their expression to achieve neuroprotection in these flies? It is conceivable that even if their transcription is increased, their protein levels remain at basal levels because of the sustained inhibition of translation present in pink1 or parkin mutants. ${ }^{16}$ Currently, this cannot be confirmed as we do not have reagents capable of detecting endogenous levels of Shmt2 or Nmdmc in flies.
We conclude that mitochondrial dysfunction caused by the loss of pink 1 and parkin in flies engages dATF4-dependent protective mechanisms. Post-mitotic cells such as neurons are reported to lack the de novo nucleotide biosynthetic pathways and rely instead on the salvage pathway; ${ }^{20}$ however, both Shmt2 and Nmdmc are components of one-carbon metabolism involved in the de novo biosynthetic pathway. Therefore, our observation that the overexpression of either of these genes can suppress neurodegeneration in fly models of PDs challenges the notion that de novo nucleotide biosynthetic pathways are irrelevant in postmitotic cells such as neurons.

In conclusion, we propose mitochondrial one-carbon metabolism is critical in at least two separate settings: it not only ensures the accurate nuclear DNA replication in proliferating cells such as cancer or embryonic cells but also counteracts mitochondrial dysfunction in post-mitotic cells such as neurons.

\section{Materials and Methods}

Genetics and Drosophila strains. Fly stocks and crosses were maintained on standard cornmeal agar media at $25^{\circ} \mathrm{C}$. The strains used were pink $1^{B 9}$, park ${ }^{25}$ and daGAL4 (kind gifts from A Whitworth, MRC, Centre for Developmental and Biomedical Genetics, University of Sheffield, Sheffield, UK), $w^{1118}$, elavGAL4 (Bloomington Stock Centre, Bloomington, IN, USA) and RNAi lines: Shmt2 (RNAi\#1, ID: 19206; RNAi\#2, ID: 19208), Nmdmc (RNAi\#1, ID: 5706; RNAi\#2, ID: 110198), dAtt4 (RNAi\#1, ID: 2935; RNAi\#2, ID: 109014) and dGcn2 (ID: 103976) (Vienna Drosophila RNAi Centre, Vienna, Austria). UAS dAtf4 was from Zurich ORFeome Project (Zurich, Switzerland) (ID: F000106). HA-tagged cDNA fragments encoding full-length Shmt2 (ID: RH67089) and Nmdmc (ID: GH01066) from Drosophila Genomics Resource Center (Bloomington, IN, USA) were cloned into pUASTattB vector for PhiC31-mediated site-directed transgenesis. Transgenic flies were generated at the Cambridge fly facility, Department of Genetics, University of Cambridge (Cambridge, UK). All the experiments on adult flies were performed with males.

Metabolic profiling. Global metabolic profiles were obtained from 3-day-old flies using the Metabolon Platform (Metabolon Inc., Morrisville, NC, USA) as previously described. ${ }^{5}$ Essentially, each sample consisted of 8 biological replicates (100 flies per replicate). The sample preparation process was carried out using the automated MicroLab STAR system from Hamilton Company (Bonaduz, GR, Switzerland). For sample extraction, $80 \%$ (v/v) methanol/water solution was used. Samples were then prepared for the appropriate instrument, either LC/MS or GC/ MS. Compounds above the detection threshold were identified by comparison with library entries of purified standards or recurrent unknown entities. Identification of known chemical entities was based on comparison with metabolomic library entries of purified standards.

Microarray acquisition and analysis. RNA was prepared from the heads of male adult flies ( 6 samples in total, 3 replicates for each genotype). The RNA quality was confirmed using an Agilent 2100 Bioanalyzer (Agilent Technologies, Santa Clara, CA, USA). Detailed experimental protocols and raw data were deposited in ArrayExpress under accession E-MTAB-1406. Differential expression was analysed using the Partek Genomics Suite (Partek Inc., St. Louis, Missouri, USA).

Pathway analysis. Data were processed using the Ingenuity Pathway Analysis (IPA), build 313398M, content version 18841524 (QIAGEN, Hilden, Germany). To determine the putative modulators of the observed expression changes in pink $1^{B 9}$ and park ${ }^{25}$ mutant flies, we employed the IPA Comparison Analysis using the Upstream Regulator analytic sub-routine. This programme computes two statistical measures for each potential transcriptional regulator (TR): an overlap $P$-value and an activation $z$-score. The overlap $P$-value calls likely upstream regulators based on significant overlap between data set genes and known targets regulated by a TR. The activation $z$-score is used to infer likely activation states of upstream regulators based on comparison with a model that assigns random regulation directions. ${ }^{14}$ In practice, $z$-scores greater than 2 or smaller than -2 can be considered significant. 
a

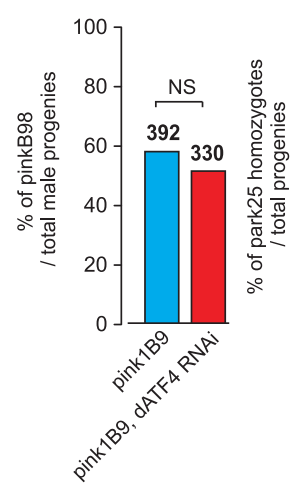

e

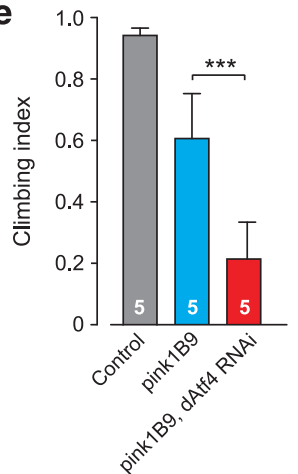

i

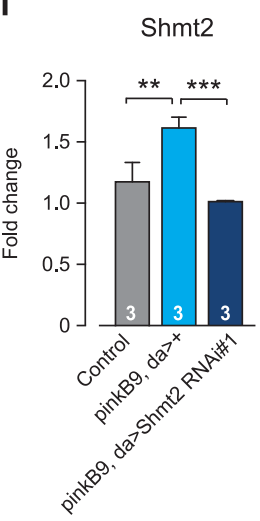

b

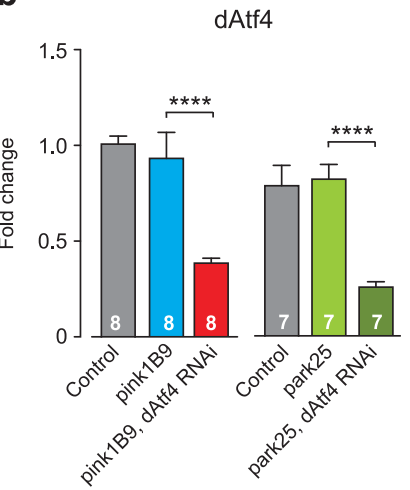

c

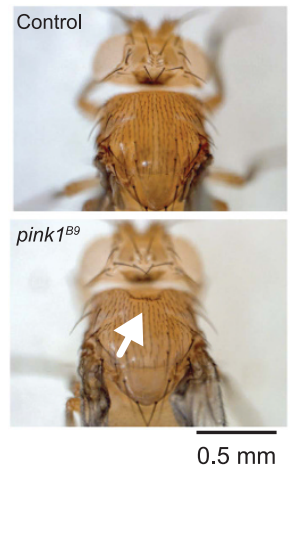

f

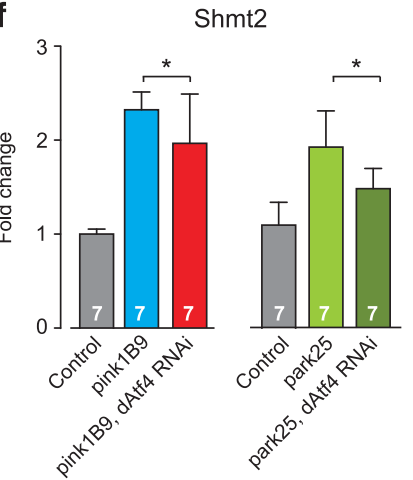

g

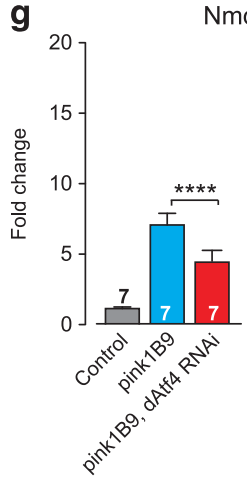

Nmdmc

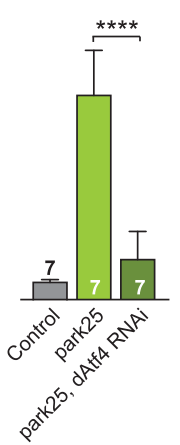

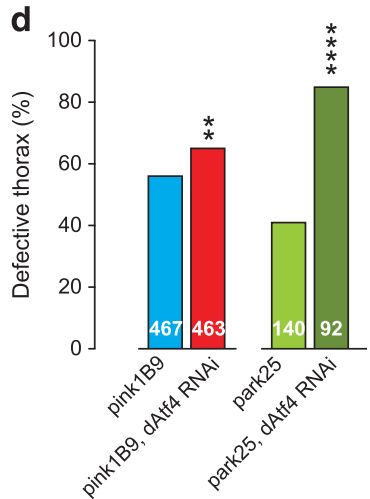

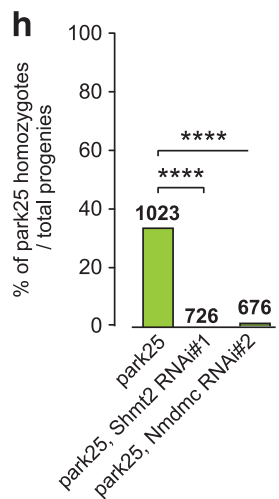

$\mathbf{k}$

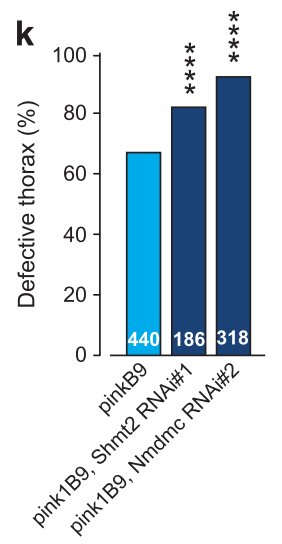

|

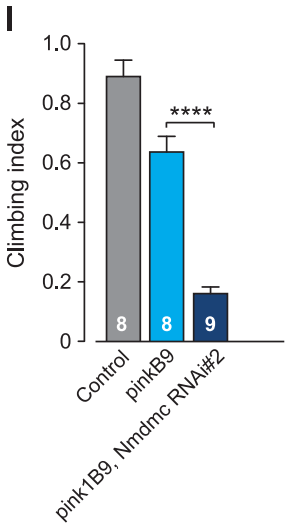

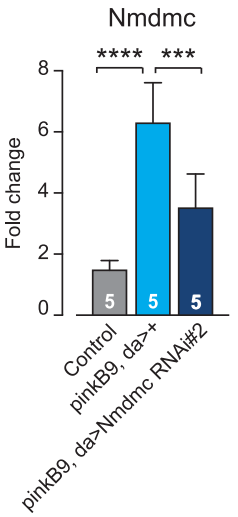

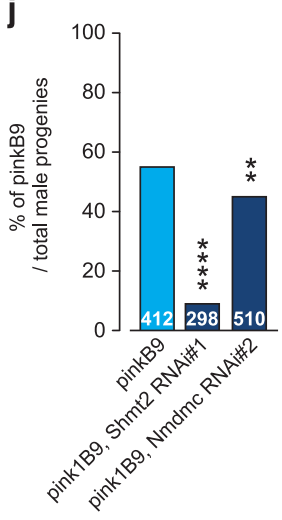

Figure 5 Suppressing the upregulation of Shmt2 or Nmdmc deteriorates the phenotype of pink1 or parkin mutants. (a) RNAi-mediated suppression of dAtt4 enhances lethality in parkin and pink1 mutants (asterisks, $\chi^{2}$ two-tailed, 95\% confidence intervals). (b) RNAi-mediated suppression of dAtf4 in pink1 or parkin mutants. Expression levels were measured by real-time qPCR (mean \pm S.D.). The significance is indicated (asterisks, two-tailed unpaired $t$-test). (c) Representative images of normal and defective thorax in pink1 mutant, arrow points to a thoracic defect. (d) Enhancement of the thoracic defects in pink1 or parkin mutants by RNAi-mediated suppression of $d A t t 4$ (asterisks, $\chi^{2}$ twotailed, $95 \%$ confidence intervals). (e) Enhancement of the motor impairment of pink1 mutants by RNAi-mediated suppression of $d A t t 4$. Flies were tested using a standard climbing assay (mean \pm S.D.; asterisks, one-way ANOVA with Bonferroni's multiple comparison test). (f and $\mathbf{g}$ ) RNAi-mediated suppression of $d A$ Atf4 in pink1 or parkin mutants affects the transcript levels of $S h m t 2(\mathbf{f})$ and $N m d m c(\mathbf{g})$. Expression levels were measured by real-time qPCR (mean \pm S.D.). The significance is indicated $(P$-value, two-tailed unpaired $t$-test compared with pink1B9 or park25). (h) RNAi-mediated suppression of Shmt2 or Nmdmc enhances lethality in parkin mutants (asterisks, $\chi^{2}$ two-tailed, $95 \%$ confidence intervals). (i) RNAi-mediated suppression of Shmt2 or Nmdmc in pink1 mutants. Expression levels were measured by real-time qPCR (mean \pm S.D.). The significant values relative to the control are indicated (asterisks, two-tailed unpaired $t$-test compared with Control). (j) RNAi-mediated suppression of Shmt2 or Nmdmc enhances lethality in pink1 mutants (asterisks, $\chi^{2}$ two-tailed, $95 \%$ confidence intervals). (k) Enhancement of the thoracic defects of pink1 mutants by RNAi-mediated suppression of Shmt2 or Nmdmc (asterisks, $\chi^{2}$ two-tailed, $95 \%$ confidence intervals). (I) Enhancement of the motor impairment of pink1 mutants by RNAi-mediated suppression of Nmdmc. Flies were tested using a standard climbing assay (mean \pm S.D.; asterisks, one-way ANOVA with Bonferroni's multiple comparison test). Genotypes in (a and b and d-I): Control: daGAL4; park25: park ${ }^{25}$, daGAL4/ park ${ }^{25}$; pink1B9: pink $1^{B 9}$, daGAL4. All RNAi lines were driven by daGAL4. (c): Control: $w^{1118}$ 
For the identification of coordinate changes in metabolite abundance in Shmt2 and Nmdmc RNAi strains, we employed the IPA Comparison Analysis workflow. This programme computes a $P$-value (Fisher's exact test) based on significant overlap between the observed metabolic changes and known canonical pathways defined by the HumanCyc metabolic pathway information data set (QIAGEN).

Cell culture and transfections. SH-SY5Y were cultured in DMEM/F12 1:1 (Gibco BRL, Waltham, MA, USA) supplemented with 10\% heat-inactivated FCS (Invitrogen, Waltham, MA, USA), $100 \mathrm{U} / \mathrm{ml}$ penicillin (Gibco BRL) and $100 \mu \mathrm{g} / \mathrm{ml}$ streptomycin (Gibco BRL). The cells were maintained at $37^{\circ} \mathrm{C}$ in $5 \% \mathrm{CO}_{2}$ in culture medium. RNA silencing was performed by delivering siRNA at a concentration of $10 \mathrm{nM}$ into SH-SY5Y cells. Lipofectamine RNAiMAX (Invitrogen) was used as the transfection reagent. The siRNA was obtained from Dharmacon (Lafayette, CO, USA) (ON-TARGETplus Non-targeting Pool, \#D-001810-10-05; ATF4, SMARTpool: ON-TARGETplus L-005125-00-0005).

Protein extraction and western blotting. Protein extracts from whole flies were prepared by grinding flies in lysis buffer $(100 \mathrm{mM} \mathrm{KCl}, 20 \mathrm{mM}$ Hepes at $\mathrm{pH} 7.5,5 \%$ (v/v) glycerol, $10 \mathrm{mM}$ EDTA, 0.1\% (v/v) Triton X-100, $10 \mathrm{mM}$ DTT, $1 \mu \mathrm{g} / \mathrm{ml}$ leupeptin, $1 \mu \mathrm{g} / \mathrm{ml}$ antipain, $1 \mu \mathrm{g} / \mathrm{ml}$ chymostatin and $1 \mu \mathrm{g} / \mathrm{ml}$ pepstatin). Protein extracts from cells were prepared by scraping them in lysis buffer $(20 \mathrm{mM}$ Tris at $\mathrm{pH} 7.5,150 \mathrm{mM} \mathrm{NaCl}, 1 \%$ (v/v) Nonidet-40, $0.5 \%$ (w/v) sodium deoxycholate, $0.1 \%(\mathrm{w} / \mathrm{v})$ SDS) containing phosphatase inhibitor cocktail tablets PhosSTOP (Roche, Basel, Switzerland) and the protease inhibitors leupeptin, antipain, chymostatin and pepstatin (Sigma, Dorset, UK) at the manufacturer's recommended dilution. The suspensions were cleared by centrifugation at $21000 \times \mathrm{g}$ for $10 \mathrm{~min}$ at $4{ }^{\circ} \mathrm{C}$ and protein concentrations of the supernatants were measured using the Bradford assay (Bio-Rad, Hercules, CA, USA). For the dATF4 immunoblot, protein extracts from whole flies were prepared by grinding flies in the same buffer as those for protein extraction from cells. All supernatants were mixed with $4 \times$ LDS loading buffer. For SDS-PAGE, equivalent amounts of proteins were resolved on $4-12 \%$ or $10 \%$ Precast Gels (Invitrogen) and transferred onto PVDF membranes (Millipore, Watford, UK). The membranes were blocked in TBS $(0.15 \mathrm{M} \mathrm{NaCl}$ and $10 \mathrm{mM}$ Tris$\mathrm{HCl}, \mathrm{pH} 7.5$ ) containing $10 \%(\mathrm{w} / \mathrm{v})$ dried non-fat milk for $1 \mathrm{~h}$ at room temperature, probed with the indicated primary antibody before being incubated with the appropriate HRP-conjugated secondary antibody. Antibody complexes were visualized by Pierce (Waltham, MA, USA) enhanced chemiluminescence (ECL).

Antibodies. Primary antibodies employed in this study were $\alpha$-tubulin (Sigma, T6074), HA (Roche Applied, Basel, Switzerland, 11583816001), ATF4 to probe human cell samples (Santa Cruz Biotechnology, Santa Cruz, CA, USA, sc-22800), dATF4 to probe Drosophila samples (a kind gift from Min Kang, University of Ulsan, Seoul, South Korea), GAPDH (Sigma, G8795), NDUFS3/complex I (Abcam, Cambridge, UK, ab14711), mtCO1 (Abcam, ab90668), $\alpha$-tubulin (Sigma, T6074) and TH (Immunostar, Hudson, WI, USA, 22941).

RNA extraction and quantitative real-time RT-PCR. Total RNA was extracted using the RNeasy Mini Kit (QIAGEN; for mammalian cells) or TRIzol (Ambion, Waltham, MA, USA; for Drosophila tissues) and quantified by spectrophotometric analysis. Quantitative real-time PCR with reverse transcription (qRTPCR) was performed on a real-time cycler (Applied Biosystems, Foster City, CA, USA, 7500 Fast Real-Time PCR Systems) using the SensiFAST SYBR Lo-ROX one-Step Kit (Bioline, London, UK). Gene-specific primers were obtained from QIAGEN (QuantiTect Primer Assays) for the following genes: DDIT3 (QT00082278), dATF4 (QT00503069), CG3999 (QT00972349), CG6415 (QT00934318), Nmdmc (dm: QT00503153, hs: QT00081592), Parkin (QT00023401), Pink1 (QT01670459) and Shmt2 (dm: QT00498904, hs: QT00012754). Gene-specific primers obtained from Sigma were: hsATF4 (forward, 5'-CCCTTCACCTTCTTACAACCTC-3'; reverse, 5'-GTCTGGCTTCCTATCTCCTTCA-3'), rp49 (forward, 5'-TGTCCTTC CAGCTTCAAGATGACCATC-3'; reverse, 5'-CTTGGGCTTGCGCCATTTGTG-3'), TBP (forward, 5'-TCAAACCCAGAATTGTTCTCCTTAT-3'; reverse, 5'-CCTGAATC CCTTTAGAATAGGGTAGA-3'). rp49 and TBP were used as housekeeping genes when analysing fly and cells samples, respectively.

Immunofluorescence and confocal microscopy. To analyse the mitochondria in larvae brains and ventral nerve cord nerves, tissues from third-instar larvae were dissected in PBS, transferred to a drop of PBS as mounting medium on glass slides, covered with coverslip and imaged on a Zeiss (Cambridge, UK) LSM510 confocal microscope.
Lifespan analysis. Groups of 15 newly eclosed males of each genotype were placed into separate vials with food and maintained at $25^{\circ} \mathrm{C}$. The flies were transferred into vials containing fresh food every 2 to 3 days, and the number of dead flies was recorded. The data are presented as Kaplan-Meier survival distributions, and the significance was determined by log-rank tests.

Climbing assay. The climbing assays were performed as previously described ${ }^{21}$ using a countercurrent apparatus. A total of 15 to 20 male flies were placed into the first chamber, tapped to the bottom and then given $20 \mathrm{~s}$ to climb a distance of $10 \mathrm{~cm}$. Those flies that successfully climbed $10 \mathrm{~cm}$ or beyond in $20 \mathrm{~s}$ were then shifted to a new chamber, and both sets of flies were given another opportunity to climb the $10 \mathrm{~cm}$ distance. This procedure was repeated a total of five times. After five trials, the number of flies in each chamber was counted. A video demonstrating the technique can be found at https://youtu.be/vmR6s_WAXgc. The climbing index was measured by using a weighted average approach using the following formula:

$\frac{(0 * n 0)+(1 * n 1)+(2 * n 2)+(3 * n 3)+(4 * n 4)+(5 * n 5)}{5 * \operatorname{SUM}(n 0 \cdot n 5)}$

In this formula, $n 0$ corresponds to the flies that fail the first trial and $n 1$ to $n 5$ the number of flies that successfully pass each respective trial. At least 100 flies were used for each genotype tested.

Drosophila phenotypic analysis. To quantify the eclosion defects, adult parent flies were removed 3 to 4 days after the setup of crosses. Eclosion of progeny was monitored for 10 days after the first fly had hatched. The total numbers of empty and full pupae cases were counted and used for statistical analysis.

To quantify lethality, the number of both male and female park ${ }^{25}$ homozygotes to total flies (park $k^{25}$ homozygotes+heterozygotes) and the number of pink $1^{19}$ to total males (pink $\left.1^{B 9}+F M 6\right)$ were counted and used for statistical analysis.

To assess downturned wing phenotype, groups of ten 3-day-old male flies were acclimatized for at least $1 \mathrm{~h}$ in fresh vials. The number of flies that presented with downturned wings was counted. Counts were repeated three times to confirm the result. The total number of flies with or without downturned wings were counted and used for statistical analysis.

Microscopy-based assessment of mitochondrial function and morphology. Measurements of $\Delta \psi \mathrm{m}$ in fly brains were performed as previously described. ${ }^{5}$ Briefly, fly brains were loaded for $40 \mathrm{~min}$ at room temperature with $40 \mathrm{nM}$ TMRM in loading buffer (10 mM HEPES pH 7.35, $156 \mathrm{mM} \mathrm{NaCl}, 3 \mathrm{mM}$ $\mathrm{KCl}, 2 \mathrm{mM} \mathrm{MgSO}_{4}, 1.25 \mathrm{mM} \mathrm{KH}_{2} \mathrm{PO}_{4}, 2 \mathrm{mM} \mathrm{CaCl}_{2}, 10 \mathrm{mM}$ glucose) and the dye was present during the experiment. In these experiments, TMRM is used in the redistribution mode to assess $\Delta \psi \mathrm{m}$, and therefore a reduction in TMRM fluorescence represents mitochondrial depolarization. Confocal images were obtained using a Zeiss 510 confocal microscope equipped with a $40 \times$ oil immersion objective. Illumination intensity was kept to a minimum (at $0.1-0.2 \%$ of laser output) to avoid phototoxicity and the pinhole was set to give an optical slice of $2 \mu \mathrm{m}$. Fluorescence was quantified by exciting TMRM using the $565 \mathrm{~nm}$ laser and measured above $580 \mathrm{~nm}$. Z-stacks of 5 fields of $300 \mu \mathrm{m}^{2}$ each per brain were acquired, and the mean maximal fluorescence intensity was measured for each group. Mitochondrial length was calculated as previously described ${ }^{22}$ by using the 'ruler' and 'measurement' tools in Photoshop CS3 Extended (San Jose, CA, USA) to measure the length of mitoGFP-positive mitochondria across their largest dimension.

Electron microscopy. For transmission electron microscopy (TEM), adult fly thoraces were fixed overnight in $0.1 \mathrm{M}$ sodium cacodylate buffer ( $\mathrm{pH} 7.4$ ) containing $2 \%$ paraformaldehyde, $2.5 \%$ glutaraldehyde and $0.1 \%$ Tween-20. Samples were postfixed for $1 \mathrm{~h}$ at room temperature in a solution containing $1 \%$ osmium tetroxide and $1 \%$ potassium ferrocyanide. After fixation, samples were stained en bloc with $5 \%$ aqueous uranyl acetate overnight at room temperature; the samples were then dehydrated via a series of ethanol washes and embedded in TAAB epoxy resin (TAAB Laboratories Equipment Ltd, Aldermaston, UK). Semi-thin sections were stained with toluidine blue, and areas of the sections were selected for ultramicrotomy. Ultrathin sections were stained with lead citrate and imaged using a MegaView 3 digital camera and iTEM software (Olympus Soft Imaging Solutions GmbH, Münster, Germany) in a Jeol 100-CXII electron microscope (Jeol UK Ltd, Welwyn Garden City, UK). 


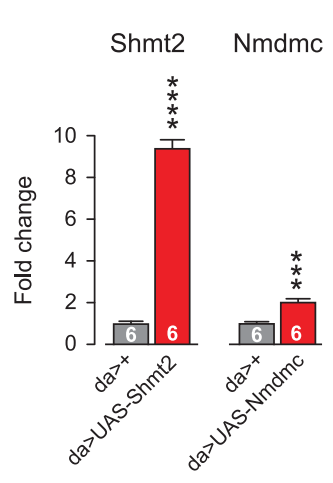

b

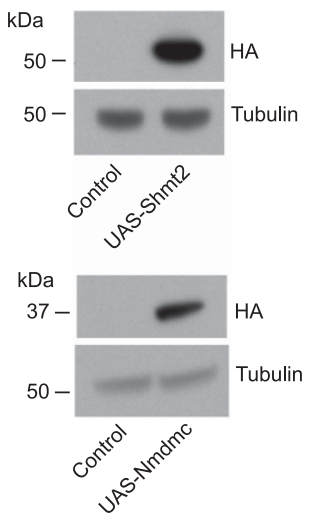

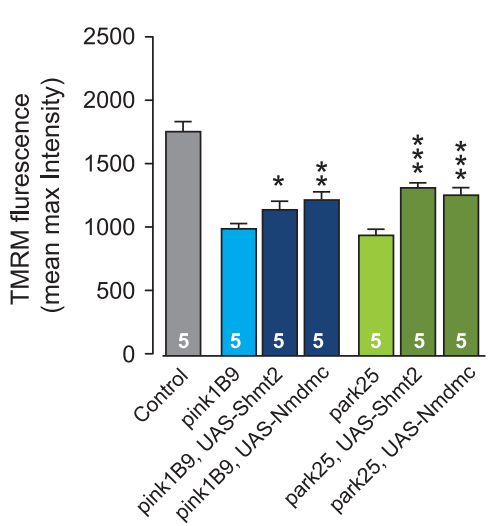

d

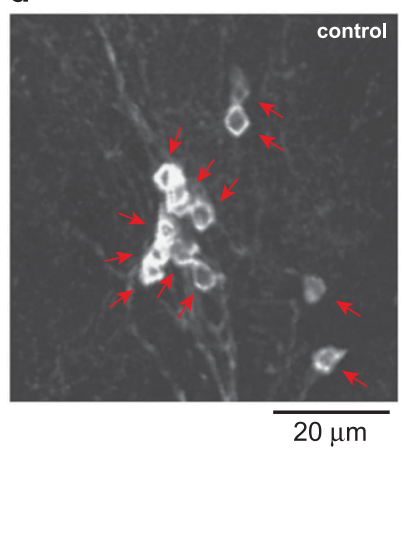

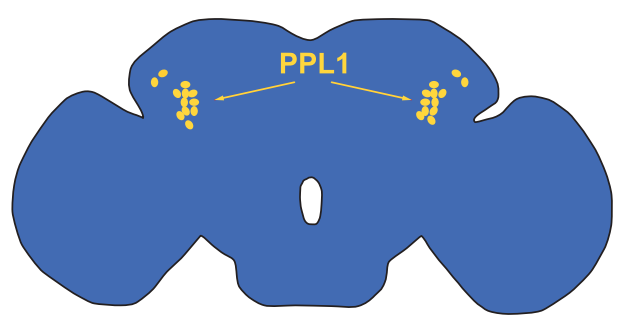

f

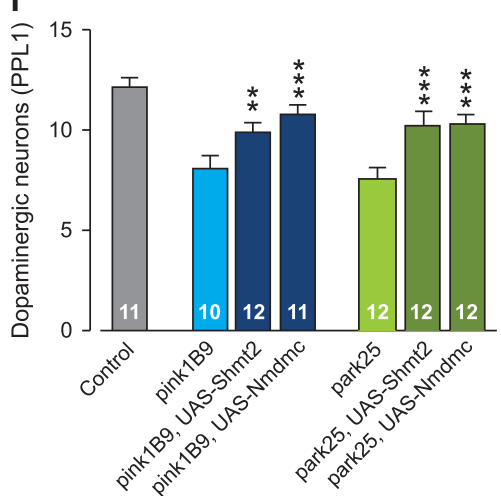

g

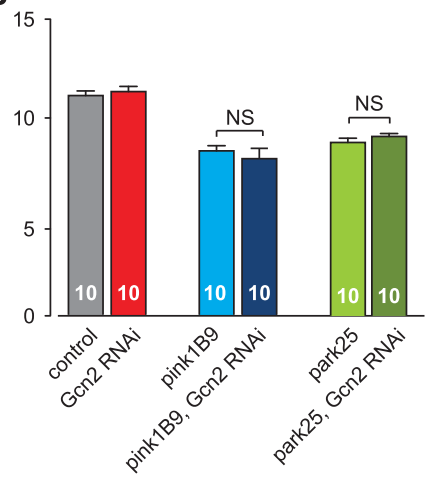

Figure 6 Neuronal loss in pink1 and parkin mutants is complemented by Shmt2 or Nmdmc. (a) Analysis of Shmt2 and Nmdmc expression levels (mean \pm S.D.; asterisks, two-tailed unpaired t-test relative to control). (b) Analysis of Shmt2 and Nmdmc protein levels. Whole-fly lysates were analysed using western blotting with the indicated antibodies. The 3-day-old flies were used for analysis of transcript and protein levels. (c) Expression of Shmt2 or Nmdmc rescues the loss of $\Delta \psi \mathrm{mm}$ (mean \pm S.D.; asterisks, oneway ANOVA with Dunnett's multiple comparison test). (d) A whole mounted control fly brain showing TH-positive neurons, arrows indicate individual PPL1 cluster neurons. (e) Schematic diagram of a fly brain in sagittal orientation indicating the PPL1 cluster of dopaminergic neurons in yellow. (f) Expression of Shmt2 or Nmdmc rescues the loss of dopaminergic neurons in the PPL1 cluster of pink1 and parkin mutant flies (mean \pm S.D.; asterisks, one-way ANOVA with Dunnett's multiple comparison test). (g) RNAi-mediated suppression of $d G c n 2$ fails to rescue the loss of dopaminergic neurons in the PPL1 cluster of pink1 or parkin mutant flies (mean \pm S.D.; asterisks, one-way ANOVA with Dunnett's multiple comparison test). Genotypes in (a and b): Control: daGAL4, Shmt2 and Nmdmc transgenes were driven by daGAL4, (c-g): Control: elavGAL4, Shmt2 and Nmdmc transgenes and RNAi dGcn2 were driven by elavGAL4

Analysis of dopaminergic neurons. Fly brains were dissected from 20-day-old flies and stained for anti-tyrosine hydroxylase (Immunostar) as previously described. ${ }^{23}$ Brains were positioned in PBS+0.1\% Triton in a coverslip clamp chamber (ALA Scientific Instruments Inc., Farmingdale, NY, USA) using a harp made of platinum wire and nylon string and imaged by confocal microscopy. Tyrosine hydroxylase-positive PPL1 cluster neurons were counted per brain hemisphere. Data acquired for the assessment of each genotype were obtained as a single experimental set before statistical analysis.

Statistical analyses. Descriptive and inferential statistical analyses were performed using GraphPad Prism 6 (www.graphpad.com). Computation of the minimal sample size for the variables measured in this study was assessed by power analysis, setting alpha initially to 0.05 , using StatMate 2 (www.graphpad.com).

Data are presented as the mean values, and the error bars indicate \pm S.D. The number of biological replicates per experimental variable $(n)$ is indicated in either the figures or figure legends. Parametric tests were used (performed using data obtained from pilot experiments) after confirming that the variables under analysis displayed Gaussian distributions using the D'Agostino-Pearson test (computed using GraphPad Prism 5). The significance is indicated as ${ }^{*} P<0.05,{ }^{* \star} P<0.01,{ }^{* \star *} P<0.001$ and ${ }^{* * \star *} P<0.0001$. For the statistical analysis of metabolites in flies, pair-wise comparisons were performed using Welch's $t$-tests. The $q$-value provides an estimate of the false discovery rate (FDR) according to Storey and Tibshirani. ${ }^{24}$ The investigators gathering quantitative data on biological samples were not blinded to the sample identities at the time of analysis. No specific randomization strategies were employed when assigning biological replicates to treatment groups.

Digital image processing. Fluorescence, transmission electron microscope and western blot images were acquired as uncompressed bitmapped digital data (TIFF format) and processed using Adobe Photoshop, employing established scientific imaging workflows. ${ }^{25}$

\section{Conflict of Interest}

The authors declare no conflict of interest.

Acknowledgements. We thank the Vienna Drosophila RNAi Center and the Bloomington Drosophila Stock Center for fly stocks; the Drosophila Genomics Resource Center for cDNA clones; the Fly Facility, Department of Genetics, University of Cambridge, for generation of transgenic lines; and J Parmar and T Ashby for fly food preparation. We thank D Dinsdale, T Smith and M Martin for assistance with Electron Microscopy. We thank Giovanna Mallucci for comments on the manuscript.

1. Kilberg MS, Shan J, Su N. ATF4-dependent transcription mediates signaling of amino acid limitation. Trends Endocrinol Metab 2009; 20: 436-443. 
2. Harding HP, Novoa I, Zhang Y, Zeng H, Wek R, Schapira M et al. Regulated translation initiation controls stress-induced gene expression in mammalian cells. Mol Cell 2000; 6 : 1099-1108.

3. Harding HP, Zhang Y, Zeng H, Novoa I, Lu PD, Calfon M et al. An integrated stress response regulates amino acid metabolism and resistance to oxidative stress. Mol Cell 2003; 11: 619-633.

4. Celardo I, Martins L, Gandhi S. Unravelling mitochondrial pathways to Parkinson's disease. Br J Pharmacol 2014; 171: 1943-1957.

5. Tufi R, Gandhi S, de Castro IP, Lehmann S, Angelova PR, Dinsdale D et al. Enhancing nucleotide metabolism protects against mitochondrial dysfunction and neurodegeneration in a PINK1 model of Parkinson's disease. Nat Cell Biol 2014; 16: 157-166.

6. Nikkanen J, Forsstrom S, Euro L, Paetau I, Kohnz RA, Wang L et al. Mitochondrial DNA replication defects disturb cellular dNTP pools and remodel one-carbon metabolism. Cell Metab 2016; 23: 635-648.

7. Tibbetts AS, Appling DR. Compartmentalization of Mammalian folate-mediated one-carbon metabolism. Annu Rev Nutr 2010; 30: 57-81.

8. Tucker EJ, Hershman SG, Kohrer C, Belcher-Timme CA, Patel J, Goldberger OA et al. Mutations in MTFMT underlie a human disorder of formylation causing impaired mitochondrial translation. Cell Metab 2011; 14: 428-434.

9. Di Pietro E, Sirois J, Tremblay ML, MacKenzie RE. Mitochondrial NAD-dependent methylenetetrahydrofolate dehydrogenase-methenyltetrahydrofolate cyclohydrolase is essential for embryonic development. Mol Cell Biol 2002; 22: 4158-4166.

10. Zhang WC, Shyh-Chang N, Yang H, Rai A, Umashankar S, Ma S et al. Glycine decarboxylase activity drives non-small cell lung cancer tumor-initiating cells and tumorigenesis. Cell 2012; 148: 259-272.

11. Jain M, Nilsson R, Sharma S, Madhusudhan N, Kitami T, Souza AL et al. Metabolite profiling identifies a key role for glycine in rapid cancer cell proliferation. Science 2012; 336: 1040-1044.

12. Lee GY, Haverty PM, Li L, Kljavin NM, Bourgon R, Lee J et al. Comparative oncogenomics identifies PSMB4 and SHMT2 as potential cancer driver genes. Cancer Res 2014; 74: 3114-3126.

13. Nilsson R, Jain M, Madhusudhan N, Sheppard NG, Strittmatter L, Kampf $C$ et al. Metabolic enzyme expression highlights a key role for MTHFD2 and the mitochondrial folate pathway in cancer. Nat Commun 2014; 5: 3128.

14. Kramer A, Green J, Pollard J Jr., Tugendreich S. Causal analysis approaches in Ingenuity Pathway Analysis. Bioinformatics 2014; 30: 523-530.

15. Jousse C, Deval C, Maurin AC, Parry L, Cherasse Y, Chaveroux $C$ et al. TRB3 inhibits the transcriptional activation of stress-regulated genes by a negative feedback on the ATF4 pathway. J Biol Chem 2007; 282: 15851-15861.
16. Celardo I, Costa AC, Lehmann S, Jones C, Wood N, Mencacci NE et al. Mitofusin-mediated ER stress triggers neurodegeneration in pink1/parkin models of Parkinson's disease. Cell Death Dis 2016; 7: e2271.

17. Lu PD, Harding HP, Ron D. Translation reinitiation at alternative open reading frames regulates gene expression in an integrated stress response. J Cell Biol 2004; 167: 27-33.

18. Vattem KM, Wek RC. Reinitiation involving upstream ORFs regulates ATF4 mRNA translation in mammalian cells. Proc Natl Acad Sci USA 2004; 101: 11269-11274.

19. Moisoi N, Klupsch K, Fedele V, East P, Sharma S, Renton A et al. Mitochondrial dysfunction triggered by loss of $\mathrm{HtrA2}$ results in the activation of a brain-specific transcriptional stress response. Cell Death Differ 2009; 16: 449-464.

20. Arner ES, Eriksson S. Mammalian deoxyribonucleoside kinases. Pharmacol Ther 1995; 67: $155-186$.

21. Greene JC, Whitworth AJ, Kuo I, Andrews LA, Feany MB, Pallanck LJ. Mitochondrial pathology and apoptotic muscle degeneration in Drosophila parkin mutants. Proc Natl Acad Sci USA 2003; 100: 4078-4083.

22. Pimenta de Castro I, Costa AC, Lam D, Tufi R, Fedele V, Moisoi N et al. Genetic analysis of mitochondrial protein misfolding in Drosophila melanogaster. Cell Death Differ 2012; 19: 1308-1316.

23. Whitworth AJ, Theodore DA, Greene JC, Benes H, Wes PD, Pallanck LJ. Increased glutathione S-transferase activity rescues dopaminergic neuron loss in a Drosophila model of Parkinson's disease. Proc Natl Acad Sci USA 2005; 102: 8024-8029.

24. Storey JD, Tibshirani R. Statistical significance for genomewide studies. Proc Natl Acad Sci USA 2003; 100: 9440-9445.

25. Wexler EJ. Photoshop CS3 Extended for Biomedical Research [DVD-ROM and online course]. Lynda.com, Inc.: Ventura, 2008.

(c) (i) () $\Theta$ This work is licensed under a Creative Commons Attribution-NonCommercial-NoDerivs 4.0 International License. The images or other third party material in this article are included in the article's Creative Commons license, unless indicated otherwise in the credit line; if the material is not included under the Creative Commons license, users will need to obtain permission from the license holder to reproduce the material. To view a copy of this license, visit http://creativecommons.org/licenses/by-nc-nd/4.0/

(C) The Author(s) 2017

Supplementary Information accompanies this paper on Cell Death and Differentiation website (http://www.nature.com/cdd) 